\title{
Traveling wave solutions of degenerate coupled multi-KdV equations
}

Cite as: J. Math. Phys. 57, 103507 (2016); https://doi.org/10.1063/1.4965444

Submitted: 08 August 2015 . Accepted: 04 October 2016 . Published Online: 24 October 2016

Metin Gürses, and Aslı Pekcan
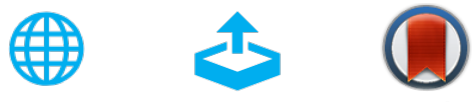

View Online

Export Citation

CrossMark

\section{ARTICLES YOU MAY BE INTERESTED IN}

Traveling wave solutions of degenerate coupled Korteweg-de Vries equation Journal of Mathematical Physics 55, 091501 (2014); https://

doi.org/10.1063/1.4893636

Darboux transformation and multi-soliton solutions of the Camassa-Holm equation and modified Camassa-Holm equation

Journal of Mathematical Physics 57, 103502 (2016); https://

doi.org/10.1063/1.4964256

A generalized Hirota-Satsuma coupled KdV system: Darboux transformations and reductions

Journal of Mathematical Physics 57, 083506 (2016); https://

doi.org/10.1063/1.4960747

\section{Journal of \\ Mathematical Physics}

Young Researcher Award

Recognizing the outstanding work of early career researchers 


\title{
Traveling wave solutions of degenerate coupled multi-KdV equations
}

\author{
Metin Gürses ${ }^{1, a)}$ and Aslı Pekcan ${ }^{2, b)}$ \\ ${ }^{1}$ Department of Mathematics, Faculty of Science, Bilkent University, 06800 Ankara, Turkey \\ ${ }^{2}$ Department of Mathematics, Faculty of Science, Hacettepe University, \\ 06800 Ankara, Turkey
}

(Received 8 August 2015; accepted 4 October 2016; published online 24 October 2016)

Traveling wave solutions of degenerate coupled $\ell-\mathrm{KdV}$ equations are studied. Due to symmetry reduction these equations reduce to one ordinary differential equation (ODE), i.e., $\left(f^{\prime}\right)^{2}=P_{n}(f)$ where $P_{n}(f)$ is a polynomial function of $f$ of degree $n=\ell+2$, where $\ell \geq 3$ in this work. Here $\ell$ is the number of coupled fields. There is no known method to solve such ordinary differential equations when $\ell \geq 3$. For this purpose, we introduce two different types of methods to solve the reduced equation and apply these methods to degenerate three-coupled KdV equation. One of the methods uses the Chebyshev's theorem. In this case, we find several solutions, some of which may correspond to solitary waves. The second method is a kind of factorizing the polynomial $P_{n}(f)$ as a product of lower degree polynomials. Each part of this product is assumed to satisfy different ODEs. Published by AIP Publishing. [http://dx.doi.org/10.1063/1.4965444]

\section{INTRODUCTION}

The system of degenerate coupled multi-field $\mathrm{KdV}$ equations is given as ${ }^{1-6}$

$$
\begin{aligned}
u_{t} & =\frac{3}{2} u u_{x}+q_{x}^{2}, \\
q_{t}^{2} & =q^{2} u_{x}+\frac{1}{2} u q_{x}^{2}+q_{x}^{3}, \\
\vdots & \vdots \quad \vdots \quad \vdots \quad \vdots \\
q_{t}^{\ell-1} & =q^{\ell-1} u_{x}+\frac{1}{2} u q_{x}^{\ell-1}+v_{x}, \\
v_{t} & =-\frac{1}{4} u_{x x x}+v u_{x}+\frac{1}{2} u v_{x},
\end{aligned}
$$

where $q^{1}=u$ and $q^{\ell}=v$. In Refs. $1-7$, it was shown that this system is also a degenerate $\mathrm{KdV}$ system of rank one. In a previous work, ${ }^{8}$ we focused on Equation (1) for $\ell=2$. We reduced this equation into an ODE, $\left(f^{\prime}\right)^{2}=P_{4}(f)$, where $P_{4}(f)$ is a polynomial function of degree four. We analyzed all possible cases about the zeros of $P_{4}(f)$. Due to this analysis, we determined the cases when the solution is periodic or solitary. When the polynomial has one double $f_{2}$ and two simple zeros $f_{1}$ and $f_{3}$ with $f_{1}<f_{2}<f_{3}$, or one triple and one simple zeros, the solution is solitary. Other cases give periodic or non-real solutions. By using the Jacobi elliptic functions, ${ }^{9}$ we obtained periodic solutions and all solitary wave solutions which rapidly decay to some constants, explicitly. We have also shown that there are no real asymptotically vanishing traveling wave solutions for $\ell=2$. Indeed, we have the following theorem for the degenerate coupled $\ell-\mathrm{KdV}$ equation, $\ell \geq 2$. The degenerate coupled $\ell-\mathrm{KdV}$ equation can be reduced to the equation

$$
\left(f^{\prime}\right)^{2}=P_{\ell+2}(f)
$$

\footnotetext{
a)gurses@fen.bilkent.edu.tr

b) aslipekcan@ @acettepe.edu.tr
} 
by taking $\ell$ functions as $u(x, t)=q^{1}(x, t)=f(\xi), q^{2}(x, t)=f_{2}(\xi), \ldots, v(x, t)=q^{\ell}=f_{\ell}(\xi)$, where $\xi=x-c t$ in (1). Here $P_{\ell+2}(f)$ is a polynomial of $f$ of degree $\ell+2$. If we apply the asymptotically vanishing boundary conditions to (2), we have

$$
\left(f^{\prime}\right)^{2}=B f^{2}(f+2 c)^{\ell} .
$$

Theorem 1.1. When $\ell=$ odd, we have $B>0$, and then the degenerate coupled $\ell-K d V$ equation has a real traveling wave solution with asymptotically vanishing boundary conditions, but when $\ell=$ even, the constant $B<0$. Hence the equation does not have a real traveling wave solution with asymptotically vanishing boundary conditions.

Proof. Consider the degenerate coupled $\ell$-KdV equation (1). Let $u(x, t)=q^{1}(x, t)=f(\xi)$, $q^{2}(x, t)=f_{2}(\xi), \ldots, v(x, t)=q^{\ell}=f_{\ell}(\xi)$, where $\xi=x-c t$. By using the first $\ell-1$ equations, we obtain all functions $f_{i}(\xi), i=2,3, \ldots, \ell$ as a polynomial of $f(\xi)$. We get

$$
\begin{aligned}
f_{2}(\xi) & =Q_{2}(f)=-c f-\frac{3}{4} f^{2}+d_{1}=-\alpha_{2} f^{2}+A_{1}(f), \\
f_{3}(\xi) & =Q_{3}(f)=\frac{3}{2} c f^{2}+\frac{1}{2} f^{3}+\left(c^{2}-d_{1}\right) f+d_{2} \\
& =\alpha_{3} f^{3}+A_{2}(f), \\
f_{4}(\xi) & =Q_{4}(f)=-\frac{5}{16} f^{4}-\frac{3}{2} c f^{3}+\left(-\frac{9}{4} c^{2}+\frac{3}{4} d_{1}\right) f^{2}+\left(-c^{3}+c d_{1}-d_{2}\right) f+d_{3} \\
& =\alpha_{4} f^{4}+A_{3}(f), \\
& \vdots \\
f_{\ell}(\xi) & =Q_{\ell}(f)=(-1)^{\ell+1} \alpha_{\ell} f^{\ell}+A_{\ell-1}(f),
\end{aligned}
$$

where $\alpha_{j}>0$ are constants, and $A_{i}(f)$ and $Q_{j}(f)$ are polynomials of $f$ of degree $i$ and $j, i=$ $1,2, \ldots, \ell-1$ and $j=2,3, \ldots, \ell$, respectively.

Now use the $\ell$ th equation. We obtain

$\frac{1}{4} f^{\prime \prime \prime}=(-1)^{\ell-1} \alpha_{\ell} f^{\prime} f^{\ell}\left(1+\frac{\ell}{2}\right)+c(-1)^{\ell-1} \ell \alpha_{\ell} f^{\prime} f^{\ell-1}+f^{\prime} A_{\ell-1}+\frac{1}{2} f \frac{\partial A_{\ell-1}(f)}{\partial x}-\frac{\partial A_{\ell-1}(f)}{\partial t}$.

Integrating the above equation once, we get

$$
\frac{1}{4} f^{\prime \prime}=(-1)^{\ell-1} \frac{\alpha_{\ell}}{\ell+1} f^{\ell+1}\left(1+\frac{\ell}{2}\right)+R_{\ell}(f) .
$$

By using $f^{\prime}$ as an integrating factor, we integrate once more. Finally, we obtain

$$
\left(f^{\prime}\right)^{2}=(-1)^{\ell-1} \frac{8 \alpha_{\ell}}{(\ell+1)(\ell+2)} f^{\ell+2}\left(1+\frac{\ell}{2}\right)+R_{\ell+1}(f)=B f^{\ell+2}+R_{\ell+1}(f),
$$

where $R_{i}(f)$ is a polynomial of $f$ of degree $i, i=\ell, \ell+1$. When $\ell$ is odd, the coefficient of $f^{\ell+2}$, that is $B$, is positive, and when $\ell$ is even, $B$ is negative. Applying asymptotically vanishing boundary conditions, we get $\left(f^{\prime}\right)^{2}=B f^{2}(f+2 c)^{\ell}$, where $\operatorname{sign}(B)=(-1)^{\ell-1}$. Hence for $\ell=$ odd, the degenerate coupled $\ell-\mathrm{KdV}$ equation has a real traveling wave solution with asymptotically vanishing boundary conditions, but when $\ell=$ even, it does not.

In this work, we study Equation (1) for $\ell=3$, which is

$$
\begin{aligned}
u_{t} & =\frac{3}{2} u u_{x}+v_{x}, \\
v_{t} & =v u_{x}+\frac{1}{2} u v_{x}+\omega_{x}, \\
\omega_{t} & =-\frac{1}{4} u_{x x x}+\omega u_{x}+\frac{1}{2} u \omega_{x},
\end{aligned}
$$

in detail. It is clear from Theorem 1.1 that unlike the case $\ell=2$, we have the real traveling wave solution with asymptotically vanishing boundary conditions in $\ell=3$ case. 
Here the system (6)-(8) reduces to a polynomial of degree five

$$
\left(f^{\prime}\right)^{2}=\frac{f^{5}}{2}+3 c f^{4}+\left(6 c^{2}-2 d_{1}\right) f^{3}+4\left(c^{3}-c d_{1}+d_{2}\right) f^{2}+8 d_{3} f+8 d_{4}=P_{5}(f),
$$

where $f(\xi)=u(x, t)$, and $c, d_{1}, d_{2}, d_{3}, d_{4}$ are constants. When the degree of the polynomial in the reduced equation is equal to five or greater, it is almost impossible to solve them. As far as we know, there is no known method to solve these equations. We shall introduce two methods to solve such equations. The first one is based on the Chebyshev's theorem ${ }^{10}$ which is used recently to solve the Einstein field equations for a cosmological model..$^{11,12}$ By using the Chebyshev's theorem, we give several solutions of the reduced equations for $\ell=3$ and also for arbitrary $\ell$. The second method is based on factorizing the polynomial $P_{\ell+2}(f)$ as the product of lower degree polynomials. In this way, we make use of the reduced equations of lower degrees. We have given all possible such solutions for $\ell=3$.

The layout of our paper is as follows: In Sec. II, we study the behavior of the solutions in the neighborhood of the zeros of $P_{5}(f)$ and discuss the cases giving solitary wave solutions. In Sec. III, we find exact solutions of the system (6)-(8) by a new method proposed for any $\ell \geq 3$ which uses the Chebyshev's theorem and analyze the cases in which we may have solitary wave and kink-type solutions. In Sec. IV, we present an alternative method which is based on a factorization of polynomials. Particularly, we find the solutions of the system (6)-(8). Here we obtain many solutions for $\ell=3$ including solitary wave, kink-type, periodic, and unbounded solutions. We present some of them in the text but the rest of the solutions are given in Appendices A and B.

\section{GENERAL WAVES OF PERMANENT FORM FOR $(\ell=3)$}

\section{A. Zeros of $P_{5}(f)$ and types of solutions}

Here we will analyze the zeros of $P_{5}(f)$ in (9).

(i) If $f_{1}=f\left(\xi_{1}\right)$ is a simple zero of $P_{5}(f)$, we have $P_{5}\left(f_{1}\right)=0$. Taylor expansion of $P_{5}(f)$ about $f_{1}$ gives

$$
\begin{aligned}
\left(f^{\prime}\right)^{2} & =P_{5}\left(f_{1}\right)+P_{5}^{\prime}\left(f_{1}\right)\left(f-f_{1}\right)+O\left(\left(f-f_{1}\right)^{2}\right) \\
& =P_{5}^{\prime}\left(f_{1}\right)\left(f-f_{1}\right)+O\left(\left(f-f_{1}\right)^{2}\right) .
\end{aligned}
$$

From here, we get $f^{\prime}\left(\xi_{1}\right)=0$ and $f^{\prime \prime}\left(\xi_{1}\right)=P_{5}^{\prime}\left(f_{1}\right) / 2$. Hence we can write the function $f(\xi)$ as

$$
\begin{aligned}
f(\xi) & =f\left(\xi_{1}\right)+\left(\xi-\xi_{1}\right) f^{\prime}\left(\xi_{1}\right)+\frac{1}{2}\left(\xi-\xi_{1}\right)^{2} f^{\prime \prime}\left(\xi_{1}\right)+O\left(\left(\xi-\xi_{1}\right)^{3}\right) \\
& =f_{1}+\frac{1}{4}\left(\xi-\xi_{1}\right)^{2} P_{5}^{\prime}\left(f_{1}\right)+O\left(\left(\xi-\xi_{1}\right)^{3}\right) .
\end{aligned}
$$

Thus, in the neighborhood of $\xi=\xi_{1}$, the function $f(\xi)$ has a local minimum or maximum as $P_{5}^{\prime}\left(f_{1}\right)$ is positive or negative, respectively, since $f^{\prime \prime}\left(\xi_{1}\right)=P_{5}^{\prime}\left(f_{1}\right) / 2$.

(ii) If $f_{1}=f\left(\xi_{1}\right)$ is a double zero of $P_{5}(f)$, we have $P_{5}\left(f_{1}\right)=P_{5}^{\prime}\left(f_{1}\right)=0$. Taylor expansion of $P_{5}(f)$ about $f_{1}$ gives

$$
\begin{aligned}
\left(f^{\prime}\right)^{2} & =P_{5}\left(f_{1}\right)+P_{5}^{\prime}\left(f_{1}\right)\left(f-f_{1}\right)+\frac{1}{2}\left(f-f_{1}\right)^{2} P_{5}^{\prime \prime}\left(f_{1}\right)+O\left(\left(f-f_{1}\right)^{3}\right) \\
& =\frac{1}{2}\left(f-f_{1}\right)^{2} P_{5}^{\prime \prime}\left(f_{1}\right)+O\left(\left(f-f_{1}\right)^{3}\right) .
\end{aligned}
$$

To have the real solution $f$, we should have $P_{5}^{\prime \prime}\left(f_{1}\right)>0$. From the equality (11), we get

which gives

$$
f^{\prime} \pm \frac{1}{\sqrt{2}} f \sqrt{P_{5}^{\prime \prime}\left(f_{1}\right)} \sim \pm \frac{1}{\sqrt{2}} f_{1} \sqrt{P_{5}^{\prime \prime}\left(f_{1}\right)}
$$

$$
f(\xi) \sim f_{1}+\alpha e^{ \pm \frac{1}{\sqrt{2}} \sqrt{P_{5}^{\prime \prime}\left(f_{1}\right) \xi}},
$$


where $\alpha$ is a constant. Hence $f \rightarrow f_{1}$ as $\xi \rightarrow \mp \infty$. The solution $f$ can have only one peak and the wave extends from $-\infty$ to $\infty$.

(iii) If $f_{1}=f\left(\xi_{1}\right)$ is a triple zero of $P_{5}(f)$, we have $P_{5}\left(f_{1}\right)=P_{5}^{\prime}\left(f_{1}\right)=P_{5}^{\prime \prime}\left(f_{1}\right)=0$. Taylor expansion of $P_{5}(f)$ about $f_{1}$ gives

$$
\begin{aligned}
\left(f^{\prime}\right)^{2} & =P_{5}\left(f_{1}\right)+P_{5}^{\prime}\left(f_{1}\right)\left(f-f_{1}\right)+\frac{1}{2}\left(f-f_{1}\right)^{2} P_{5}^{\prime \prime}\left(f_{1}\right)+\frac{1}{6}\left(f-f_{1}\right)^{3}+O\left(\left(f-f_{1}\right)^{4}\right) \\
& =\frac{1}{6}\left(f-f_{1}\right)^{3} P_{5}^{\prime \prime \prime}\left(f_{1}\right)+O\left(\left(f-f_{1}\right)^{4}\right) .
\end{aligned}
$$

This is valid only if both signs of $\left(f-f_{1}\right)^{3}$ and $P_{5}^{\prime \prime \prime}\left(f_{1}\right)$ are same. Hence, to obtain the real solution $f$ we have the following two possibilities:

(1) $\left(f-f_{1}\right)>0$ and $P_{5}^{\prime \prime \prime}\left(f_{1}\right)>0$,

(2) $\left(f-f_{1}\right)<0$ and $P_{5}^{\prime \prime \prime}\left(f_{1}\right)<0$.

If $\left(f-f_{1}\right)>0$ and $P_{5}^{\prime \prime \prime}\left(f_{1}\right)>0$, then we have

$$
f^{\prime} \sim \pm \frac{1}{\sqrt{6}}\left(f-f_{1}\right)^{3 / 2} \sqrt{P_{5}^{\prime \prime \prime}\left(f_{1}\right)},
$$

which gives

$$
f(\xi) \sim f_{1}+\frac{4}{\left( \pm \frac{1}{\sqrt{6}} \sqrt{P_{5}^{\prime \prime \prime}\left(f_{1}\right)} \xi+\alpha_{1}\right)^{2}},
$$

where $\alpha_{1}$ is a constant. Thus $f \rightarrow f_{1}$ as $\xi \rightarrow \pm \infty$.

Let $\left(f-f_{1}\right)<0$ and $P_{5}^{\prime \prime \prime}\left(f_{1}\right)<0$. Then

$$
f^{\prime} \sim \pm \frac{1}{\sqrt{6}}\left(f_{1}-f\right)^{3 / 2} \sqrt{-P_{5}^{\prime \prime \prime}\left(f_{1}\right)},
$$

which yields

$$
f(\xi) \sim f_{1}-\frac{4}{\left( \pm \frac{1}{\sqrt{6}} \sqrt{-P_{5}^{\prime \prime \prime}\left(f_{1}\right)} \xi+\alpha_{2}\right)^{2}},
$$

where $\alpha_{2}$ is a constant. Thus $f \rightarrow f_{1}$ as $\xi \rightarrow \pm \infty$.

(iv) If $f_{1}=f\left(\xi_{1}\right)$ is a quadruple zero of $P_{5}(f)$, then we have $P_{5}\left(f_{1}\right)=P_{5}^{\prime}\left(f_{1}\right)=P_{5}^{\prime \prime}\left(f_{1}\right)=$ $P_{5}^{\prime \prime \prime}\left(f_{1}\right)=0$. In this case, Taylor expansion of $P_{5}(f)$ about $f_{1}$ gives

$$
\left(f^{\prime}\right)^{2}=\frac{1}{24}\left(f-f_{1}\right)^{4} P_{5}^{(4)}\left(f_{1}\right)+O\left(\left(f-f_{1}\right)^{5}\right) .
$$

This is valid only if $P_{5}^{(4)}\left(f_{1}\right)>0$. Then we have

$$
f^{\prime} \sim \pm \frac{1}{2 \sqrt{6}}\left(f-f_{1}\right)^{2} \sqrt{P_{5}^{(4)}\left(f_{1}\right)},
$$

which gives

$$
f(\xi) \sim f_{1}-\frac{1}{ \pm \frac{1}{2 \sqrt{6}} \sqrt{P_{5}^{(4)}\left(f_{1}\right)} \xi+\gamma_{1}},
$$

where $\gamma_{1}$ is a constant. Thus $f \rightarrow f_{1}$ as $\xi \rightarrow \pm \infty$.

(v) If $f_{1}=f\left(\xi_{1}\right)$ is a zero of multiplicity 5 of $P_{5}(f)$, then we have $P_{5}(f)=\left(f-f_{1}\right)^{5} / 2$. This is valid only if $f-f_{1}>0$. So we obtain the solution $f$ as

$$
f=f_{1}+\left(\frac{4}{9}\right)^{1 / 3} \frac{1}{\left( \pm \frac{\xi}{\sqrt{2}}+m_{1}\right)^{2 / 3}},
$$

where $m_{1}$ is a constant. Hence $f \rightarrow f_{1}$ as $\xi \rightarrow \pm \infty$. 


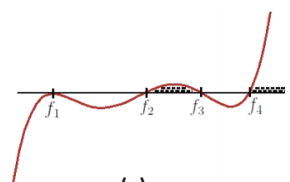

(a)

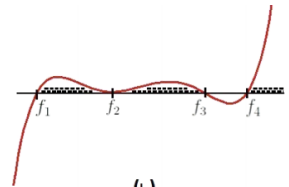

(b)

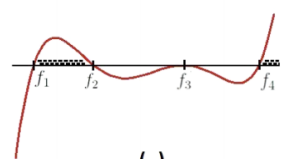

$(c)$

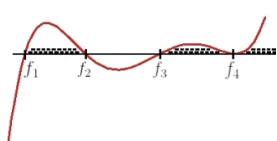

(d)

FIG. 1. Graphs of $P_{5}(f)$ having one double and three simple zeros.

\section{B. All possible cases giving solitary wave solutions}

We analyze all possible cases about the zeros of $P_{5}(f)$ that may give solitary wave solutions. Here in each cases, we will present the sketches of the graphs of $P_{5}(f)$. Real solutions of $\left(f^{\prime}\right)^{2}=P_{5}(f) \geq 0$ occur in the shaded regions.

\section{One double and three simple zeros}

In Figure $1(\mathrm{~b}), f_{1}, f_{3}$, and $f_{4}$ are simple zeros and $f_{2}$ is a double zero. The real solution occurs when $f$ stays between $f_{1}$ and $f_{2}$ or $f_{2}$ and $f_{3}$. At $f_{1}, P_{5}^{\prime}\left(f_{1}\right)=f^{\prime \prime}\left(\xi_{1}\right)>0$, hence the graph of the function $f$ is concave up at $\xi_{1}$. At double zero $f_{2}, f \rightarrow f_{2}$ as $\xi \rightarrow \pm \infty$. Hence we have a solitary wave solution with amplitude $f_{1}-f_{2}<0$. Similarly at $f_{3}, P_{5}^{\prime}\left(f_{3}\right)=f^{\prime \prime}\left(\xi_{3}\right)<0$, hence the graph of the function $f$ is concave down at $\xi_{3}$. Therefore, we also have a solitary wave solution with amplitude $f_{3}-f_{2}>0$.

Now consider the graph (d) in Figure 1. For $f_{3}$ we have $P_{5}^{\prime}\left(f_{3}\right)=f^{\prime \prime}\left(\xi_{3}\right)>0$, thus the graph of the function is concave up at $\xi_{3}$. At double zero $f_{4}, f \rightarrow f_{4}$ as $\xi \rightarrow \pm \infty$. Hence we have a solitary wave solution with amplitude $f_{3}-f_{4}<0$. In other cases, we have periodic solutions.

\section{Two double and one simple zeros}

In Figure 2(f), $f_{1}$ and $f_{3}$ are double zeros and $f_{2}$ is a simple zero. The real solution occurs when $f$ stays between $f_{2}$ and $f_{3}$. For $f_{2}$, we have $P_{5}^{\prime}\left(f_{2}\right)=f^{\prime \prime}\left(\xi_{2}\right)>0$, thus the graph of the function is concave up at $\xi_{2}$. At double zero $f_{3}, f \rightarrow f_{3}$ as $\xi \rightarrow \pm \infty$. Hence we have a solitary wave solution with amplitude $f_{2}-f_{3}<0$.

Now consider the graph $(\mathrm{g})$ in Figure 2. Here $f_{2}$ and $f_{3}$ are double zeros and $f_{1}$ is a simple zero. The real solution occurs when $f$ stays between $f_{1}$ and $f_{2}$ or $f_{2}$ and $f_{3}$. For $f_{1}$ we have $P_{5}^{\prime}\left(f_{1}\right)=f^{\prime \prime}\left(\xi_{1}\right)>0$, thus the graph of the function is concave up at $\xi_{1}$. At double zero $f_{2}, f \rightarrow f_{2}$ as $\xi \rightarrow \pm \infty$. Hence we have a solitary wave solution with amplitude $f_{1}-f_{2}<0$. The other cases give kink, anti-kink type, or unbounded solutions.

\section{One triple and two simple zeros}

Consider the graph (h) in Figure 3. Here $f_{2}$ and $f_{3}$ are simple zeros and $f_{1}$ is a triple zero. The real solution occurs when $f$ stays between $f_{1}$ and $f_{2}$. For $f_{2}$ we have $P_{5}^{\prime}\left(f_{2}\right)=f^{\prime \prime}\left(\xi_{2}\right)<0$, so the graph of the function $f$ is concave down at $\xi_{2}$. At triple zero $f_{1}, f \rightarrow f_{1}$ as $\xi \rightarrow \pm \infty$. Hence we may have a solitary wave solution with amplitude $f_{2}-f_{1}>0$.

In the graph in Figure 3(i), $f_{1}$ and $f_{3}$ are simple zeros and $f_{2}$ is a triple zero. The real solution occurs when $f$ stays between $f_{1}$ and $f_{2}$. For $f_{1}$ we have $P_{5}^{\prime}\left(f_{1}\right)=f^{\prime \prime}\left(\xi_{1}\right)>0$, so the graph of the

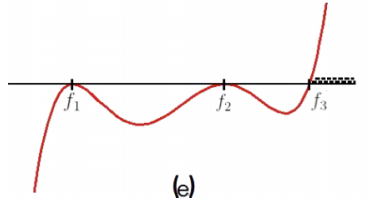

(e)

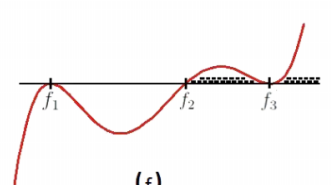

$(f)$

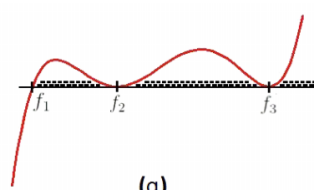

(g)

FIG. 2. Graphs of $P_{5}(f)$ having two double and one simple zeros. 


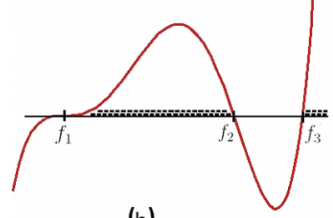

(h)

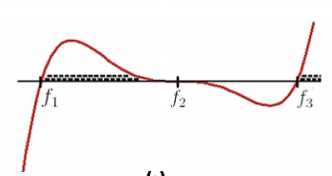

(i)

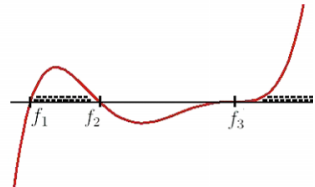

(j)

FIG. 3. Graphs of $P_{5}(f)$ having one triple and two simple zeros.

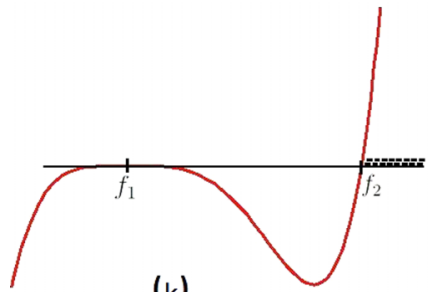

(k)

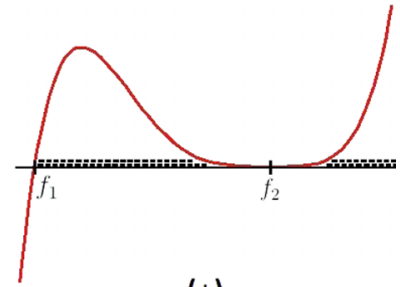

(1)

FIG. 4. Graphs of $P_{5}(f)$ having one quadruple and one simple zeros.
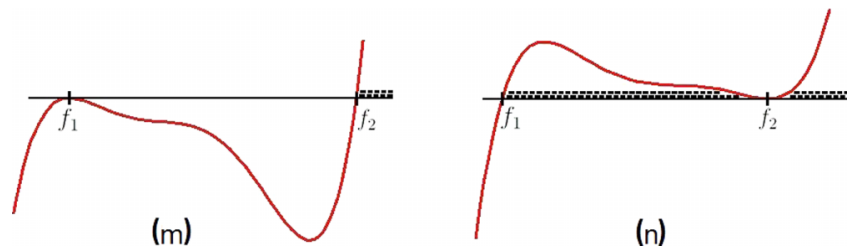

FIG. 5. Graphs of $P_{5}(f)$ having one double and one simple zeros.

function $f$ is concave up at $\xi_{1}$. At triple zero $f_{2}, f \rightarrow f_{2}$ as $\xi \rightarrow \pm \infty$. Hence we may have a solitary wave solution with amplitude $f_{1}-f_{2}<0$. The other case gives a periodic solution.

\section{One quadruple and one simple zeros}

In the graph in Figure 4(1), $f_{1}$ is a simple zero and $f_{2}$ is a quadruple zero. The real solution occurs between $f_{1}$ and $f_{2}$. At $f_{1}$ we have $P_{5}^{\prime}\left(f_{1}\right)=f^{\prime \prime}\left(\xi_{1}\right)>0$, so the graph of the function $f$ is concave up at $\xi_{1}$. At quadruple zero $f_{2}, f \rightarrow f_{2}$ as $\xi \rightarrow \pm \infty$. Hence we may have a solitary wave solution with amplitude $f_{1}-f_{2}<0$. The other case gives unbounded solution.

\section{One double and one simple zeros}

Consider the graph in Figure 5(n). Here $f_{1}$ is a simple zero and $f_{2}$ is a double zero. The real solution occurs between $f_{1}$ and $f_{2}$. At $f_{1}$ we have $P_{5}^{\prime}\left(f_{1}\right)=f^{\prime \prime}\left(\xi_{1}\right)>0$, so the graph of the function $f$ is concave up at $\xi_{1}$. At double zero $f_{2}, f \rightarrow f_{2}$ as $\xi \rightarrow \pm \infty$. Hence we have a solitary wave solution with amplitude $f_{1}-f_{2}<0$. The other case gives an unbounded solution.

To sum up, we can give the following proposition for $\ell=3$ case.

Proposition 2.1. Equation (9) may admit solitary wave solutions when the polynomial function $P_{5}(f)$ admits (i) one double and three simple zeros, (ii) two double and one simple zeros, (iii) one triple and two simple zeros, (iv) one quadruple and one simple zeros, and (v) one double and one simple zeros.

\section{EXACT SOLUTIONS BY USING THE CHEBYSHEV'S THEOREM}

The Chebyshev's theorem is given as follows. ${ }^{10}$ 
Theorem 3.1. Let $a, b, c, \alpha$, and $\beta$ be the given real numbers and $\alpha \beta \neq 0$. The antiderivative,

$$
I=\int x^{a}\left(\alpha+\beta x^{b}\right)^{c} d x
$$

is expressible by means of the elementary functions only in the three cases

$$
\text { (1) } \frac{a+1}{b}+c \in \mathbb{Z}, \quad \text { (2) } \quad \frac{a+1}{b} \in \mathbb{Z}, \quad \text { (3) } \quad c \in \mathbb{Z} .
$$

The term $x^{a}\left(\alpha+\beta x^{b}\right)^{c}$ is called a differential binomial. Note that the differential binomial may be expressed in terms of the incomplete beta function and the hypergeometric function. Let us define $u=\beta x^{b} / \alpha$. Then we have

$$
\begin{aligned}
I & =\frac{1}{b} \alpha^{\frac{a+1}{b}+c} \beta^{-\frac{a+1}{b}} B_{y}\left(\frac{1+a}{b}, c-1\right) . \\
& =\frac{1}{1+a} \alpha^{\frac{a+1}{b}+c} \beta^{-\frac{a+1}{b}} u^{\frac{1+a}{b}} F\left(\frac{a+1}{b}, 2-c ; \frac{1+a+b}{b} ; u\right),
\end{aligned}
$$

where $B_{y}$ is the incomplete beta function and $F(\tau, \kappa ; \eta ; u)$ is the hypergeometric function. Our aim is to transform the system (1) to $\left(y^{\prime}\right)^{2}=\bar{P}_{\ell+2}(y)$ by taking $f(\xi)=\gamma+\bar{\alpha} y(\bar{\beta} \xi)$. We can apply the Chebyshev's theorem to this equation, if we assume that $\bar{P}_{\ell+2}(y)$ reduces to the form $\bar{P}_{\ell+2}(y)=$ $A y^{-2 a}\left(\alpha+\beta y^{b}\right)^{-2 c}$, where $-2 a-2 c+b=\ell+2$. For $\ell=3$, let $u(x, t)=f(\xi)=\gamma+\bar{\alpha} y(\bar{\beta} \xi)$ in (9), then the equation becomes

$$
\left(y^{\prime}\right)^{2}=\bar{P}_{5}(y)=\alpha_{1} y^{5}+\alpha_{2} y^{4}+\alpha_{3} y^{3}+\alpha_{4} y^{2}+\alpha_{5} y+\alpha_{6},
$$

where

$$
\begin{aligned}
& \alpha_{1}=\frac{\bar{\alpha}^{3}}{2 \bar{\beta}^{2}}, \\
& \alpha_{2}=\frac{1}{\bar{\beta}^{2}}\left(\frac{5 \bar{\alpha}^{2} \gamma}{2}+3 \bar{\alpha}^{2} c\right), \\
& \alpha_{3}=\frac{1}{\bar{\beta}^{2}}\left(5 \bar{\alpha} \gamma^{2}+6 \bar{\alpha} c^{2}-2 \bar{\alpha} d_{1}+12 \bar{\alpha} c \gamma\right), \\
& \alpha_{4}=\frac{1}{\bar{\beta}^{2}}\left(-4 c d_{1}+4 d_{2}+18 c \gamma^{2}-6 d_{1} \gamma+5 \gamma^{3}+4 c^{3}+18 c^{2} \gamma\right), \\
& \alpha_{5}=\frac{1}{\bar{\alpha} \bar{\beta}^{2}}\left(8 d_{3}+12 c \gamma^{3}+\frac{5 \gamma^{4}}{2}+18 c^{2} \gamma^{2}-8 c d_{1} \gamma+8 c^{3} \gamma+8 d_{2} \gamma-6 d_{1} \gamma^{2}\right), \\
& \alpha_{6}=\frac{1}{\bar{\alpha}^{2} \bar{\beta}^{2}}\left(8 d_{3} \gamma+6 c^{2} \gamma^{3}+\frac{\gamma^{5}}{2}+8 d_{4}-4 c d_{1} \gamma^{2}-2 d_{1} \gamma^{3}+4 c^{3} \gamma^{2}+4 d_{2} \gamma^{2}\right) .
\end{aligned}
$$

To apply the Chebyshev's theorem 3.1 we assume that $\bar{P}_{5}(y)$ reduces to the following form:

$$
\bar{P}_{5}(y)=C y^{-2 a}\left(\alpha+\beta y^{b}\right)^{-2 c},
$$

where $a, b$, and $c$ are the constants in Theorem $3.1, b-2 a-2 c=5$, and $C$ is a constant.

Here we present the cases mentioned in Proposition 2.1. Other cases are given in Appendix A.

(1) Let $\bar{P}_{5}(y)=y\left(\alpha+\beta y^{2}\right)^{2}$. This form corresponds to the case of one simple or one simple and two double zeros. We have $y^{\prime}= \pm y^{1 / 2}\left(\alpha+\beta y^{2}\right)$, so

$$
\int y^{-1 / 2}\left(\alpha+\beta y^{2}\right)^{-1} d y= \pm \xi+A
$$

Here $a=-1 / 2, b=2$, and $c=-1$. Hence
(1) $\frac{a+1}{b}+c=-3 / 4 \notin \mathbb{Z}$,
(2) $\frac{a+1}{b}=1 / 4 \notin \mathbb{Z}$,
(3) $c=-1 \in \mathbb{Z}$. 


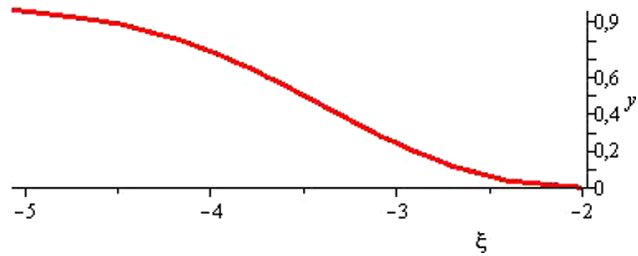

FIG. 6. Graph of a solution of $\left(y^{\prime}\right)^{2}=y\left(-1+y^{2}\right)^{2}$.

For (3), from (24), by letting $u=\beta y^{b} / \alpha$, we obtain

$$
2 \alpha^{-3 / 4} \beta^{-1 / 4} u^{1 / 4} F\left(\frac{1}{4}, 3 ; \frac{5}{4} ; u\right)= \pm \xi+A .
$$

Here choosing $\alpha=-1, \beta=1$, the integration constant $A=2$, and the plus sign in (24), we have

$$
\operatorname{arctanh}(\sqrt{y})+\arctan (\sqrt{y})=-(\xi+2)
$$

and the graph of this solution for $\xi \leq-2$ is given in Figure 6.

This is a kink-type solution.

(2) Let $\bar{P}_{5}(y)=y(\alpha+\beta y)^{4}$. This form corresponds to the case of one simple and one quadruple zeros. We have $y^{\prime}= \pm y^{1 / 2}(\alpha+\beta y)^{2}$, so

$$
\int y^{-1 / 2}(\alpha+\beta y)^{-2} d y= \pm \xi+A .
$$

Here $a=-1 / 2, b=1$, and $c=-2$. Hence
(1) $\frac{a+1}{b}+c=-3 / 2 \notin \mathbb{Z}$,
(2) $\frac{a+1}{b}=1 / 2 \notin \mathbb{Z}$,
(3) $c=-2 \in \mathbb{Z}$.

For (3), from (27), by letting $u=\beta y^{b} / \alpha$, we obtain

$$
2 \alpha^{-3 / 2} \beta^{-1 / 2} u^{1 / 2} F\left(\frac{1}{2}, 4 ; \frac{3}{2} ; u\right)=\frac{\sqrt{y}}{\alpha(\beta y+\alpha)}+\frac{\arctan \left(\frac{\beta \sqrt{y}}{\sqrt{\beta \alpha}}\right)}{\alpha \sqrt{\beta \alpha}}= \pm \xi+A .
$$

Here choosing $\alpha=1, \beta=1$, the integration constant $A=2$, and the plus sign in (27), we have

$$
\frac{\sqrt{y}}{1+y}+\arctan (\sqrt{y})=\xi+2
$$

and the graph of this solution for $-2 \leq \xi<\pi / 2-2$ is given in Figure 7 .

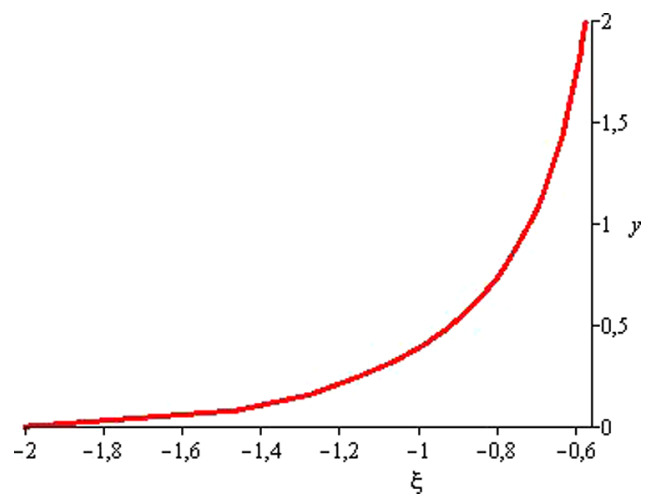

FIG. 7. Graph of a solution of $\left(y^{\prime}\right)^{2}=y(1+y)^{4}$. 
(3) Let $\bar{P}_{5}(y)=y^{2}\left(\alpha+\beta y^{3}\right)$. This form corresponds to the case of one simple and one double zeros. We have $y^{\prime}= \pm y\left(\alpha+\beta y^{3}\right)^{1 / 2}$, so

$$
\int y^{-1}\left(\alpha+\beta y^{3}\right)^{-1 / 2} d y= \pm \xi+A
$$

Here $a=-1, b=3$, and $c=-1 / 2$. Hence
(1) $\frac{a+1}{b}+c=-1 / 2 \notin \mathbb{Z}$,
(2) $\frac{a+1}{b}=0 \in \mathbb{Z}$
(3) $c=-1 / 2 \notin \mathbb{Z}$.

For (2), from (30), we obtain

$$
y=\left(\frac{\alpha}{\beta}\left(\tanh ^{2}\left(\frac{3}{2} \sqrt{\alpha}(A \pm \xi)\right)-1\right)\right)^{1 / 3} .
$$

Here choosing $\alpha=4, \beta=4$, the integration constant $A=2$, and the plus sign in (30), we obtain

$$
y(\xi)=-(\operatorname{sech}(3 \xi+6))^{2 / 3}
$$

and the graph of this solution is given in Figure 8.

This is clearly a solitary wave solution.

(4) Let $\bar{P}_{5}(y)=y^{2}(\alpha+\beta y)^{3}$. This form corresponds to the case of one double and one triple zeros. We have $y^{\prime}= \pm y(\alpha+\beta y)^{3 / 2}$, so

$$
\int y^{-1}(\alpha+\beta y)^{-3 / 2} d y= \pm \xi+A .
$$

Here $a=-1, b=1$, and $c=-3 / 2$. Hence
(1) $\frac{a+1}{b}+c=-3 / 2 \notin \mathbb{Z}$,
(2) $\frac{a+1}{b}=0 \in \mathbb{Z}$
(3) $c=-3 / 2 \notin \mathbb{Z}$.

For (2), from (33), we obtain

$$
\frac{2}{\alpha \sqrt{\beta y+\alpha}}-\frac{2 \operatorname{arctanh}\left(\frac{\sqrt{\beta y+\alpha}}{\sqrt{\alpha}}\right)}{\alpha^{3 / 2}}= \pm \xi+A .
$$

Here choosing $\alpha=1, \beta=1, A=2$, and the plus sign in (33), we have

$$
\frac{2}{\sqrt{y+1}}-2 \operatorname{arctanh}(\sqrt{y+1})=\xi+2
$$

and the graph of this solution is given in Figure 9.

(5) Let $\bar{P}_{5}(y)=y^{4}(\alpha+\beta y)$. This form corresponds to the case of one simple and one quadruple zeros. We have $y^{\prime}= \pm y^{2}(\alpha+\beta y)^{1 / 2}$, so

$$
\int y^{-2}(\alpha+\beta y)^{-1 / 2} d y= \pm \xi+A \text {. }
$$

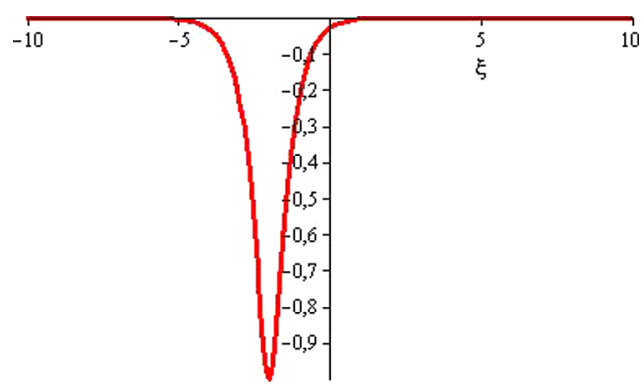

FIG. 8. Graph of a solution of $\left(y^{\prime}\right)^{2}=y^{2}\left(4+4 y^{3}\right)$. 


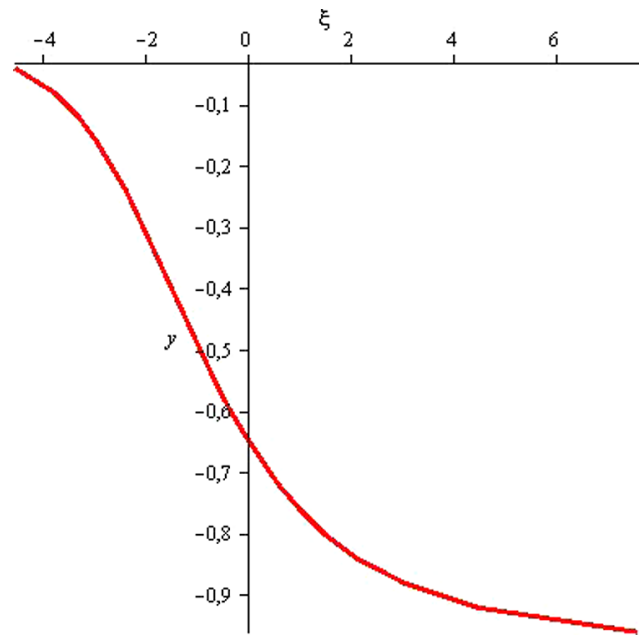

FIG. 9. Graph of a solution of $\left(y^{\prime}\right)^{2}=y^{2}(1+y)^{3}$.

Here $a=-2, b=1$, and $c=-1 / 2$. Hence
(1) $\frac{a+1}{b}+c=-3 / 2 \notin \mathbb{Z}$,
(2) $\frac{a+1}{b}=-1 \in \mathbb{Z}$,
(3) $c=-1 / 2 \notin \mathbb{Z}$.

For (2), from (36), by letting $u=\beta y^{b} / \alpha$, we obtain

$$
-\alpha^{-3 / 2} \beta u^{-1} F\left(-1, \frac{5}{2} ; 0 ; u\right)=-\frac{\sqrt{\beta y+\alpha}}{\alpha y}+\frac{\beta \operatorname{arctanh}\left(\frac{\sqrt{\beta y+\alpha}}{\sqrt{\alpha}}\right)}{\alpha^{3 / 2}}= \pm \xi+A .
$$

Taking $\alpha=1, \beta=1, A=2$, and the plus sign in (36), we get

$$
\operatorname{arctanh}(\sqrt{y+1})-\frac{\sqrt{y+1}}{y}=\xi+2
$$

and the graph of this solution for $\xi \geq-2$ is given in Figure 10 .

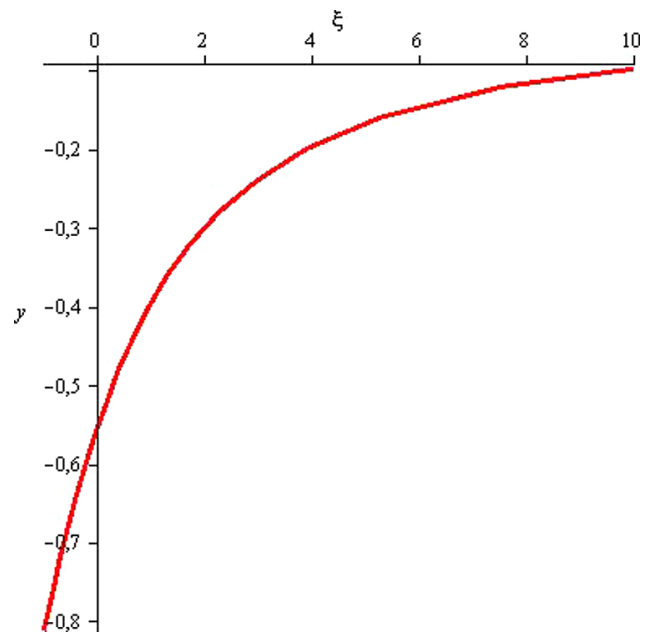

FIG. 10. Graph of a solution of $\left(y^{\prime}\right)^{2}=y^{4}(1+y)$. 


\section{AN ALTERNATIVE METHOD TO SOLVE $\left(f^{\prime}\right)^{2}=P_{n}(f)$, WHEN $n \geq 5$ : FACTORIZATION OF POLYNOMIALS}

When we have $\left(f^{\prime}\right)^{2}=P_{n}(f)$ for the reduced equation, it becomes quite difficult to solve such equations for $n \geq 5$. For this purpose, we shall introduce a new method which is based on the factorization of the polynomial $P_{n}(f)=P_{\ell+2}(f), n \geq 5$. Let the polynomial $P_{\ell+2}(f)$ have $\ell+2$ real roots, i.e., $P_{\ell+2}(f)=B \prod_{i=1}^{\ell+2}\left(f-f_{i}\right), B$ is a constant. Define a new function $\rho(\xi)$, so that $f=f(\rho(\xi))$. Hence we have $\left(f^{\prime}\right)^{2}=\left(\frac{d f}{d \rho}\right)^{2}\left(\frac{d \rho}{d \xi}\right)^{2}$. By taking

$$
\begin{aligned}
& \left(\frac{d f}{d \rho}\right)^{2}=\kappa \prod_{i=1}^{N_{1}}\left(f-f_{i}\right), \\
& \left(\frac{d \rho}{d \xi}\right)^{2}=\mu \prod_{i=1}^{N_{2}}\left(f-f_{i}\right),
\end{aligned}
$$

where $N_{1}+N_{2}=\ell+2$ and $B=\kappa \mu$, we get a system of ordinary differential equations. Solving this system gives the solution of the degenerate coupled $\ell$-KdV equation.

For illustration, we start with a differential equation where we know the solution. Consider

$$
\left(y^{\prime}\right)^{2}=\alpha\left(y-y_{1}\right)\left(y-y_{2}\right)\left(y-y_{3}\right)\left(y-y_{4}\right) .
$$

Let $y=y(\rho(\xi))$ so

$$
\left(\frac{d y}{d \rho}\right)^{2}\left(\frac{d \rho}{d \xi}\right)^{2}=\alpha\left(y-y_{1}\right)\left(y-y_{2}\right)\left(y-y_{3}\right)\left(y-y_{4}\right)
$$

Taking

$$
\begin{aligned}
& \left(\frac{d y}{d \rho}\right)^{2}=\kappa\left(y-y_{1}\right)\left(y-y_{2}\right), \\
& \left(\frac{d \rho}{d \xi}\right)^{2}=\mu\left(y-y_{3}\right)\left(y-y_{4}\right),
\end{aligned}
$$

where $\kappa \mu=\alpha$. We start with solving Equation (40). We have

$$
\frac{d y}{\sqrt{\left(y-y_{1}\right)\left(y-y_{2}\right)}}=\frac{d y}{b \sqrt{\left(\frac{y-a}{b}\right)^{2}-1}}= \pm \sqrt{\kappa} d \rho,
$$

where $a=\left(y_{1}+y_{2}\right) / 2$ and $b=\left(y_{1}-y_{2}\right) / 2$. Let $(y-a) / b=\cosh z$. This gives us $d z= \pm \sqrt{\kappa} d \rho$. After taking the integral, we obtain

$$
\operatorname{arccosh}\left(\frac{y-a}{b}\right)= \pm \sqrt{\kappa} \rho+C_{1}, \quad C_{1} \quad \text { constant }
$$

so that

$$
y=h(\rho)=a+b \cosh \left(\sqrt{\kappa} \rho+C_{2}\right), \quad C_{2} \text { constant. }
$$

Now we insert this solution into Eq. (41). Let $\cosh \left(\sqrt{\kappa} \rho+C_{2}\right)=v$. Hence we get

$$
\int \frac{d v}{\sqrt{\kappa} b \sqrt{v^{2}-1} \sqrt{(v-A)^{2}-B^{2}}}= \pm \sqrt{\mu} \xi+C_{3},
$$

where $A=\left(y_{3}+y_{4}-y_{1}-y_{2}\right) /\left(y_{1}-y_{2}\right), B=\left(y_{3}-y_{4}\right) /\left(y_{1}-y_{2}\right)$, and $C_{3}$ is a constant. Solving (43) and using (42) gives

$$
y=\frac{y_{1}\left(y_{3}-y_{2}\right)+y_{2}\left(y_{1}-y_{3}\right) \operatorname{sn}^{2}\left((1 / 2) \sqrt{\kappa\left(y_{3}-y_{2}\right)\left(y_{4}-y_{1}\right)}\left(\sqrt{\mu} \xi+C_{3}\right), k\right)}{\left(y_{3}-y_{2}\right)+\left(y_{1}-y_{3}\right) \operatorname{sn}^{2}\left((1 / 2) \sqrt{\kappa\left(y_{3}-y_{2}\right)\left(y_{4}-y_{1}\right)}\left(\sqrt{\mu} \xi+C_{3}\right), k\right)},
$$

which was obtained in Ref. 8. Here $s n$ is the Jacobi elliptic function and $k$ is the elliptic modulus satisfying $k^{2}=\left(f_{2}-f_{4}\right)\left(f_{1}-f_{3}\right) /\left(f_{2}-f_{3}\right)\left(f_{1}-f_{4}\right)$. 
Now we apply our method to the case when the polynomial $P_{n}(f)$ is of degree $n=5$. We have several possible cases, but here we shall give the case when the polynomial (9) has five real roots. Other cases are presented in Appendix B.

Case 1. If (9) has five real roots, we can write it in the form

$$
\left(f^{\prime}\right)^{2}=\alpha\left(f-f_{1}\right)\left(f-f_{2}\right)\left(f-f_{3}\right)\left(f-f_{4}\right)\left(f-f_{5}\right),
$$

where $f_{1}, f_{2}, f_{3}, f_{4}$, and $f_{5}$ are the zeros of the polynomial function $P_{5}(f)$. Now define a new function $\rho(\xi)$, so that $f=f(\rho(\xi))$. Hence (45) becomes

$$
\left(f^{\prime}\right)^{2}=\left(\frac{d f}{d \rho}\right)^{2}\left(\frac{d \rho}{d \xi}\right)^{2}=\alpha\left(f-f_{1}\right)\left(f-f_{2}\right)\left(f-f_{3}\right)\left(f-f_{4}\right)\left(f-f_{5}\right) .
$$

(i) Take

$$
\begin{aligned}
& \left(\frac{d f}{d \rho}\right)^{2}=-\left(f-f_{1}\right)\left(f-f_{2}\right)\left(f-f_{3}\right)\left(f-f_{4}\right), \\
& \left(\frac{d \rho}{d \xi}\right)^{2}=-\alpha\left(f-f_{5}\right) .
\end{aligned}
$$

In Ref. 8, we have found the solutions of Eq. (47). One of the solutions is

$$
f=h(\rho)=\frac{f_{4}\left(f_{3}-f_{1}\right)+f_{3}\left(f_{1}-f_{4}\right) \operatorname{sn}^{2}\left((1 / 2) \sqrt{\left(f_{1}-f_{3}\right)\left(f_{2}-f_{4}\right)} \rho, k\right)}{\left(f_{3}-f_{1}\right)+\left(f_{1}-f_{4}\right) \operatorname{sn}^{2}\left((1 / 2) \sqrt{\left(f_{1}-f_{3}\right)\left(f_{2}-f_{4}\right)} \rho, k\right)},
$$

where $k$ is the elliptic modulus satisfying $k^{2}=\left(f_{3}-f_{2}\right)\left(f_{4}-f_{1}\right) /\left(f_{3}-f_{1}\right)\left(f_{4}-f_{2}\right)$. We use this solution in Equation (48) and get

$$
\int_{0}^{\rho} \frac{d \hat{\rho}}{\sqrt{f_{5}-h(\hat{\rho})}}=\int_{0}^{\rho} \sqrt{\frac{A+B \mathrm{sn}^{2}(\omega \hat{\rho}, k)}{C+D \mathrm{sn}^{2}(\omega \hat{\rho}, k)}} d \hat{\rho}= \pm \sqrt{\alpha} \xi+C_{1}, \quad C_{1} \quad \text { constant }
$$

where

$$
A=f_{3}-f_{1}, B=f_{1}-f_{4}, C=\left(f_{5}-f_{4}\right)\left(f_{3}-f_{1}\right), D=\left(f_{5}-f_{3}\right)\left(f_{1}-f_{4}\right),
$$

and $\omega=(1 / 2) \sqrt{\left(f_{1}-f_{3}\right)\left(f_{2}-f_{4}\right)}$. For particular values, $f_{1}=f_{2}=-1, f_{3}=3, f_{4}=0, f_{5}=2, \alpha=$ $1, C_{1}=0$, and choosing the plus sign in (50), we get the graph of the solution $f(\xi)$ given in Figure 11 .

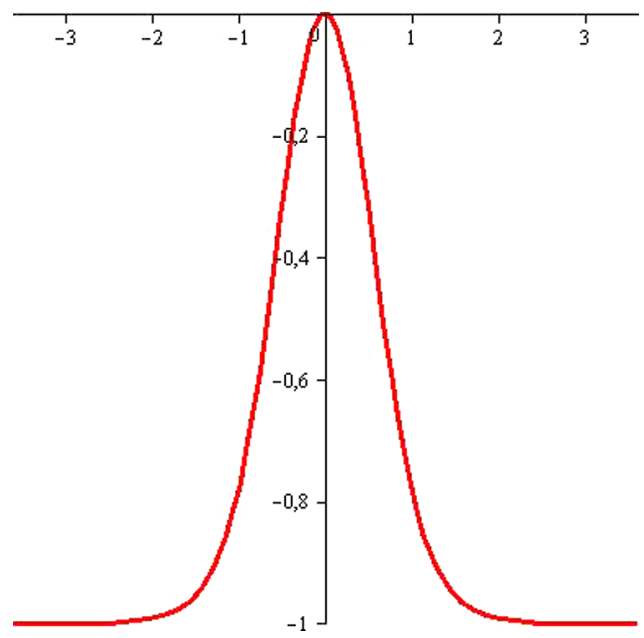

FIG. 11. Graph of the solution (49) with (50) for particular parameters. 
This is a solitary wave solution.

(ii) Take

$$
\begin{aligned}
& \left(\frac{d f}{d \rho}\right)^{2}=\kappa\left(f-f_{1}\right)\left(f-f_{2}\right)\left(f-f_{3}\right), \\
& \left(\frac{d \rho}{d \xi}\right)^{2}=\mu\left(f-f_{4}\right)\left(f-f_{5}\right),
\end{aligned}
$$

where $\kappa \mu=\alpha$. Eq. (51) has a solution

$$
f=h(\rho)=\left(f_{2}-f_{1}\right) \operatorname{sn}^{2}\left((1 / 2) \sqrt{f_{3}-f_{1}}\left(\sqrt{\kappa} \rho+C_{1}\right), k\right)+f_{1},
$$

where $k$ satisfies $k^{2}=\left(f_{1}-f_{2}\right) /\left(f_{1}-f_{3}\right)$ and $C_{1}$ is a constant. We use this solution in Eq. (52) and get

$$
\int_{0}^{\rho} \frac{d \hat{\rho}}{\sqrt{\left(h(\hat{\rho})-f_{4}\right)\left(h(\hat{\rho})-f_{5}\right)}}=\int_{0}^{\rho} \frac{d \hat{\rho}}{\sqrt{A \operatorname{sn}^{4}(\omega \hat{\rho}+\delta, k)+B \operatorname{sn}^{2}(\omega \hat{\rho}+\delta, k)+C}}= \pm \sqrt{\mu} \xi+C_{2},
$$

where $C_{2}$ is a constant and

$$
A=\left(f_{2}-f_{1}\right)^{2}, B=\left(f_{2}-f_{1}\right)\left(2 f_{1}-f_{4}-f_{5}\right), C=\left(f_{1}-f_{4}\right)\left(f_{1}-f_{5}\right), \omega=(1 / 2) \sqrt{\kappa\left(f_{3}-f_{1}\right)},
$$

and $\delta=(1 / 2) \sqrt{f_{3}-f_{1}} C_{1}$. For particular values, $f_{1}=1, f_{2}=2, f_{3}=3, f_{4}=-1, f_{5}=-2, \kappa=\mu=$ $1, C_{1}=0$, and choosing the plus sign in (54), we get the graph of the solution $f(\xi)$ given in Figure 12 .

This solution is periodic.

(iii) Take

$$
\begin{aligned}
& \left(\frac{d f}{d \rho}\right)^{2}=\kappa\left(f-f_{1}\right)\left(f-f_{2}\right), \\
& \left(\frac{d \rho}{d \xi}\right)^{2}=\mu\left(f-f_{3}\right)\left(f-f_{4}\right)\left(f-f_{5}\right),
\end{aligned}
$$

where $\kappa \mu=\alpha$. Consider first Equation (55).

We have

$$
\frac{d f}{\sqrt{\left(f-f_{1}\right)\left(f-f_{2}\right)}}= \pm \sqrt{\kappa} d \rho
$$

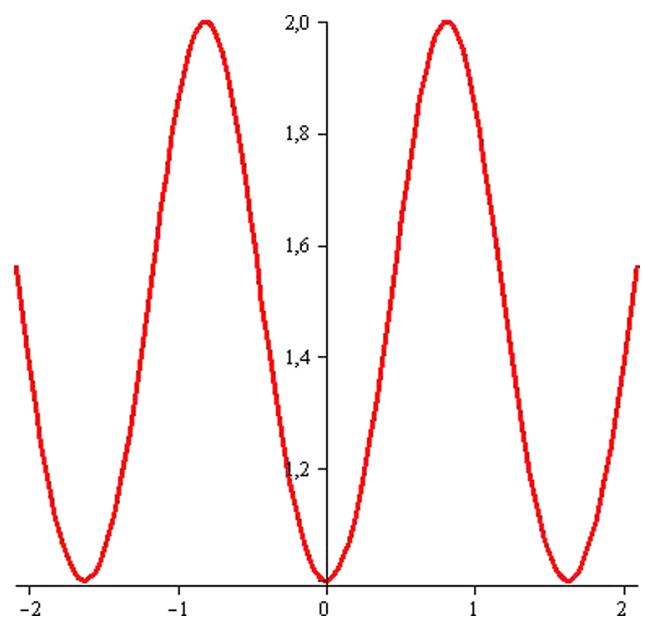

FIG. 12. Graph of the solution (53) with (54) for particular parameters. 
This equality can be reduced to

$$
\frac{d f}{b \sqrt{\left(\frac{f-a}{b}\right)^{2}-1}}= \pm \sqrt{\kappa} d \rho,
$$

where $a=\left(f_{1}+f_{2}\right) / 2$ and $b=\left(f_{1}-f_{2}\right) / 2$. By making the change of variables $(f-a) / b=u$, we get

$$
\int \frac{d u}{\sqrt{u^{2}-1}}= \pm \sqrt{\kappa} \rho+C_{1}
$$

where $C_{1}$ is a constant. Thus, we obtain

$$
\operatorname{arccosh} u=\operatorname{arccosh}\left(\frac{f-a}{b}\right)= \pm \sqrt{\kappa} \rho+C_{1},
$$

which yields

$$
f=h(\rho)=a+b \cosh \left(\sqrt{\kappa} \rho+C_{1}\right), \quad a=\left(f_{1}+f_{2}\right) / 2, \quad b=\left(f_{1}-f_{2}\right) / 2 .
$$

Now we use this result in (56),

$$
\begin{aligned}
& \int_{0}^{\rho} \frac{d \hat{\rho}}{\sqrt{\left(h(\hat{\rho})-f_{3}\right)\left(h(\hat{\rho})-f_{4}\right)\left(h(\hat{\rho})-f_{5}\right)}}= \\
= & \int_{0}^{\rho} \frac{d \hat{\rho}}{\sqrt{A \cosh ^{3}\left(\sqrt{\kappa} \hat{\rho}+C_{1}\right)+B \cosh ^{2}\left(\sqrt{\kappa} \hat{\rho}+C_{1}\right)+C \cosh \left(\sqrt{\kappa} \hat{\rho}+C_{1}\right)+D}} \\
= & \pm \sqrt{\mu} \xi+C_{2},
\end{aligned}
$$

where $C_{2}$ is a constant and

$$
A=b^{3}, B=b^{2}\left(3 a-f_{3}-f_{4}-f_{5}\right), C=b\left\{\left(a-f_{3}\right)\left(a-f_{4}\right)+\left(a-f_{3}\right)\left(a-f_{5}\right)+\left(a-f_{4}\right)\left(a-f_{5}\right)\right\},
$$
and $D=\left(a-f_{3}\right)\left(a-f_{4}\right)\left(a-f_{5}\right)$.

For particular values, $f_{1}=5, f_{2}=1, f_{3}=f_{4}=-3, f_{5}=-4, \kappa=4, \mu=1, C_{1}=C_{2}=0$, and choosing the plus sign in (62), we get the graph of the solution $f(\xi)$ given in Figure 13.

(iv) Take

$$
\begin{aligned}
& \left(\frac{d f}{d \rho}\right)^{2}=\kappa\left(f-f_{1}\right) \\
& \left(\frac{d \rho}{d \xi}\right)^{2}=\mu\left(f-f_{2}\right)\left(f-f_{3}\right)\left(f-f_{4}\right)\left(f-f_{5}\right),
\end{aligned}
$$

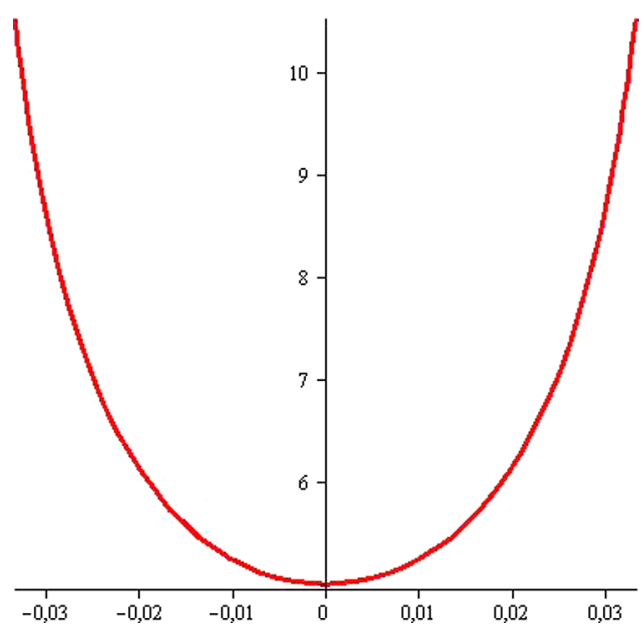

FIG. 13. Graph of the solution (61) for particular parameters 


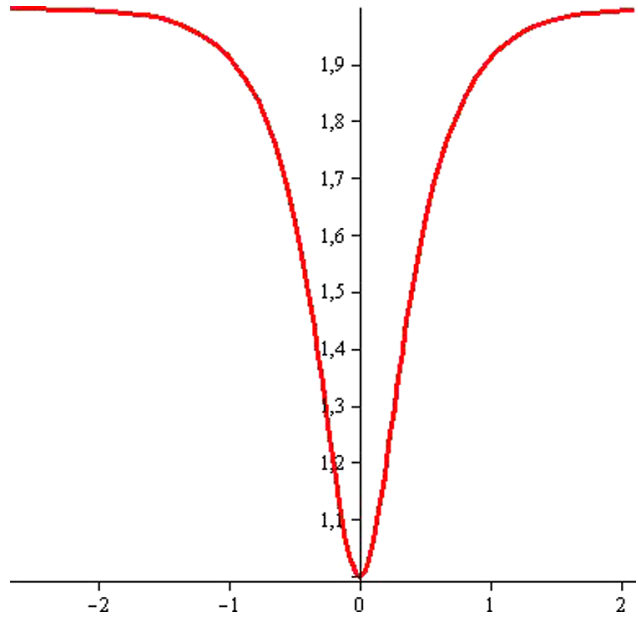

FIG. 14. Graph of the solution (66) for particular parameters.

where $\kappa \mu=\alpha$. Consider Eq. (63). We have

$$
\frac{d f}{\sqrt{f-f_{1}}}= \pm \sqrt{\kappa} d \rho
$$

Integrating both sides gives

$$
f=h(\rho)=\left( \pm \frac{\sqrt{\kappa}}{2} \rho+C_{1}\right)^{2}+f_{1}, \quad C_{1} \quad \text { constant. }
$$

We use this result in Equation (64),

$$
\begin{aligned}
& \int_{0}^{\rho} \frac{d \hat{\rho}}{\sqrt{\left(h(\hat{\rho})-f_{2}\right)\left(h(\hat{\rho})-f_{3}\right)\left(h(\hat{\rho})-f_{4}\right)\left(h(\hat{\rho})-f_{5}\right)}}= \\
& =\int_{0}^{\rho} \frac{d \hat{\rho}}{\sqrt{\left(\frac{\kappa}{4} \hat{\rho}^{2} \pm \sqrt{\kappa} C_{1} \hat{\rho}+A\right)\left(\frac{\kappa}{4} \hat{\rho}^{2} \pm \sqrt{\kappa} C_{1} \hat{\rho}+B\right)\left(\frac{\kappa}{4} \hat{\rho}^{2} \pm \sqrt{\kappa} C_{1} \hat{\rho}+C\right)\left(\frac{\kappa}{4} \hat{\rho}^{2} \pm \sqrt{\kappa} C_{1} \hat{\rho}+D\right)}} \\
& = \pm \sqrt{\mu} \xi+C_{2},
\end{aligned}
$$

where $C_{2}$ is a constant and

$$
A=C_{1}^{2}+f_{1}-f_{2}, B=C_{1}^{2}+f_{1}-f_{3}, C=C_{1}^{2}+f_{1}-f_{4}, D=C_{1}^{2}+f_{1}-f_{5} .
$$

For particular values, $f_{1}=1, f_{2}=f_{3}=2, f_{4}=3, f_{5}=4, \kappa=4, \mu=1, C_{1}=C_{2}=0$, and choosing the plus sign in (67), we get the graph of the solution $f(\xi)$ given in Figure 14.

This is a solitary wave solution.

\section{CONCLUSION}

We study the degenerate $\ell$-coupled $\mathrm{KdV}$ equations. We reduce these equations into an ODE of the form $\left(f^{\prime}\right)^{2}=P_{\ell+2}(f)$, where $P_{\ell+2}$ is a polynomial function of $f$ of degree $\ell+2, \ell \geq 3$. We give a general approach to solve the degenerate $\ell$-coupled equations by introducing two new methods that one of them uses the Chebyshev's theorem and the other one is an alternative method, based on the factorization of $P_{\ell+2}(f), \ell \geq 3$. Particularly, for the degenerate three-coupled $\mathrm{KdV}$ equations, we obtain solitary-wave, kink-type, periodic, or unbounded solutions by using these methods.

\section{ACKNOWLEDGMENTS}

This work is partially supported by the Scientific and Technological Research Council of Turkey (TÜBİTAK). 


\section{APPENDIX A: OTHER CASES USING CHEBYSHEV'S THEOREM FOR DEGENERATE THREE-COUPLED KdV EQUATION}

Here we present other cases that we use the Chebyshev's theorem to solve degenerate threecoupled $\mathrm{KdV}$ equation.

(1) Let $\bar{P}_{5}(y)=y\left(\alpha+\beta y^{4}\right)$. This form corresponds to the case of one simple zero or three simple zeros. We have $y^{\prime}= \pm y^{1 / 2}\left(\alpha+\beta y^{4}\right)^{1 / 2}$, so

$$
\int y^{-1 / 2}\left(\alpha+\beta y^{4}\right)^{-1 / 2} d y= \pm \xi+A .
$$

Here $a=-1 / 2, b=4$, and $c=-1 / 2$. So we have

$$
\text { (1) } \frac{a+1}{b}+c=-3 / 8 \notin \mathbb{Z}, \quad \text { (2) } \frac{a+1}{b}=1 / 8 \notin \mathbb{Z}, \quad \text { (3) } c=-1 / 2 \notin \mathbb{Z} .
$$

Hence we cannot obtain a solution through the Chebyshev's theorem.

(2) Let $\bar{P}_{5}(y)=y^{3}\left(\alpha+\beta y^{2}\right)$. This form corresponds to the case of one triple or two simple and one triple zeros. We have $y^{\prime}= \pm y^{3 / 2}\left(\alpha+\beta y^{2}\right)^{1 / 2}$, so

$$
\int y^{-3 / 2}\left(\alpha+\beta y^{2}\right)^{-1 / 2} d y= \pm \xi+A .
$$

Here $a=-3 / 2, b=2$, and $c=-1 / 2$. So we have
(1) $\frac{a+1}{b}+c=-3 / 4 \notin \mathbb{Z}$,
(2) $\frac{a+1}{b}=-1 / 4 \notin \mathbb{Z}$,
(3) $c=-1 / 2 \notin \mathbb{Z}$.

Hence we cannot obtain a solution through the Chebyshev's theorem.

(3) Let $\bar{P}_{5}(y)=y^{3}(\alpha+\beta y)^{2}$. This form corresponds to the case of one double and one triple zeros. We have $y^{\prime}= \pm y^{3 / 2}(\alpha+\beta y)$, so

$$
\int y^{-3 / 2}(\alpha+\beta y)^{-1} d y= \pm \xi+A .
$$

Here $a=-3 / 2, b=1$, and $c=-1$. Hence
(1) $\frac{a+1}{b}+c=-3 / 2 \notin \mathbb{Z}$,
(2) $\frac{a+1}{b}=-1 / 2 \notin \mathbb{Z}$
(3) $c=-1 \in \mathbb{Z}$.

For (3), from (A1), by letting $u=\beta y^{b} / \alpha$, we obtain

$$
-2 \alpha^{-3 / 2} \beta^{1 / 2} u^{-1 / 2} F\left(-\frac{1}{2}, 3 ; \frac{1}{2} ; u\right)=-\frac{2}{\alpha \sqrt{y}}+\frac{2 \sqrt{\beta} \arctan \left(\frac{\sqrt{\beta y}}{\sqrt{\alpha}}\right)}{\alpha^{3 / 2}}= \pm \xi+A .
$$

(4) Let $\bar{P}_{5}(y)=(\alpha+\beta y)^{5}$. This form corresponds to the case of a zero with multiplicity five. We have $y^{\prime}= \pm(\alpha+\beta y)^{5 / 2}$, so

$$
\int(\alpha+\beta y)^{-5 / 2} d y= \pm \xi+A .
$$

Here $a=0, b=1$, and $c=-5 / 2$. Hence
(1) $\frac{a+1}{b}+c=-3 / 2 \notin \mathbb{Z}$,
(2) $\frac{a+1}{b}=1 \in \mathbb{Z}$
(3) $c=-5 / 2 \notin \mathbb{Z}$.

For (2), from (A3), by letting $u=\beta y^{b} / \alpha$, we obtain

$$
\alpha^{-3 / 2} \beta^{-1} u F\left(1, \frac{9}{2} ; 2 ; u\right)=-\frac{2}{3 \beta(\beta y+\alpha)^{3 / 2}}= \pm \xi+A .
$$

Here we get

$$
y=\frac{1}{\beta}\left[\left(\frac{4}{9 \beta^{2}( \pm \xi+A)^{2}}\right)-\alpha\right] .
$$


(5) Let $\bar{P}_{5}(y)=\left(\alpha+\beta y^{5}\right)$. This form corresponds to the case of one simple zero. We have $y^{\prime}= \pm\left(\alpha+\beta y^{5}\right)^{1 / 2}$, so

$$
\int\left(\alpha+\beta y^{5}\right)^{-1 / 2} d y= \pm \xi+A .
$$

Here $a=0, b=5$, and $c=-1 / 2$. We have
(1) $\frac{a+1}{b}+c=-3 / 10 \notin \mathbb{Z}$,
(2) $\frac{a+1}{b}=1 / 5 \notin \mathbb{Z}$,
(3) $c=-1 / 2 \notin \mathbb{Z}$.

Hence we cannot obtain a solution through the Chebyshev's theorem.

\section{APPENDIX B: OTHER CASES USING ALTERNATIVE METHOD FOR DEGENERATE THREE-COUPLED KdV EQUATION}

Here we present the other cases for the method of factorization of the polynomial $P_{5}(f)$.

Case 2. If (9) has three real roots, we can write it in the form

$$
\left(f^{\prime}\right)^{2}=\alpha\left(f-f_{1}\right)\left(f-f_{2}\right)\left(f-f_{3}\right)\left(a f^{2}+b f+c\right),
$$

where $f_{1}, f_{2}$, and $f_{3}$ are the zeros of the polynomial function $P_{5}(f)$ of degree five and $b^{2}-4 a c<0$. Now define a new function $\rho(\xi)$, so that $f=f(\rho(\xi))$. Hence (B1) becomes

$$
\left(f^{\prime}\right)^{2}=\left(\frac{d f}{d \rho}\right)^{2}\left(\frac{d \rho}{d \xi}\right)^{2}=\alpha\left(f-f_{1}\right)\left(f-f_{2}\right)\left(f-f_{3}\right)\left(a f^{2}+b f+c\right) .
$$

(i) Take

$$
\begin{aligned}
& \left(\frac{d f}{d \rho}\right)^{2}=\kappa\left(f-f_{1}\right)\left(f-f_{2}\right)\left(f-f_{3}\right), \\
& \left(\frac{d \rho}{d \xi}\right)^{2}=\mu\left(a f^{2}+b f+c\right),
\end{aligned}
$$

where $\kappa \mu=\alpha$. Consider the first equation above. We have

$$
\frac{d f}{\sqrt{\left(f-f_{1}\right)\left(f-f_{2}\right)\left(f-f_{3}\right)}}= \pm \sqrt{\kappa} d \rho .
$$

We know from Case 1(ii) that Eq. (B3) has a solution

$$
f=h(\rho)=\left(f_{2}-f_{1}\right) \operatorname{sn}^{2}\left((1 / 2) \sqrt{f_{3}-f_{1}}\left(\sqrt{\kappa} \rho+C_{1}\right)\right)+f_{1},
$$

where $C_{1}$ is a constant. We use this solution in (B4), and we get

$$
\int_{0}^{\rho} \frac{d \hat{\rho}}{\sqrt{a h^{2}(\hat{\rho})+b h(\hat{\rho})+c}}=\int_{0}^{\rho} \frac{d \hat{\rho}}{\sqrt{A \operatorname{sn}^{4}(\omega \hat{\rho}+\delta)+B \operatorname{sn}^{2}(\omega \hat{\rho}+\delta)+C}}= \pm \sqrt{\mu} \xi+C_{2},
$$

where $C_{2}$ is a constant and

$$
A=a\left(f_{2}-f_{1}\right)^{2}, B=\left(2 a f_{1}+b\right)\left(f_{2}-f_{1}\right), C=\left(a f_{1}^{2}+b f_{1}+c\right), \omega=(1 / 2) \sqrt{\kappa\left(f_{3}-f_{1}\right)},
$$

and $\delta=(1 / 2) \sqrt{f_{3}-f_{1}} C_{1}$.

(ii) Take

$$
\begin{aligned}
& \left(\frac{d f}{d \rho}\right)^{2}=\kappa\left(f-f_{1}\right)\left(f-f_{2}\right), \\
& \left(\frac{d \rho}{d \xi}\right)^{2}=\mu\left(f-f_{3}\right)\left(a f^{2}+b f+c\right),
\end{aligned}
$$

where $\kappa \mu=\alpha$. Consider first Equation (B8). It has a solution

$$
f=h(\rho)=a_{1}+b_{1} \cosh \left(\sqrt{\kappa} \rho+C_{1}\right), \quad a_{1}=\left(f_{1}+f_{2}\right) / 2, \quad b_{1}=\left(f_{1}-f_{2}\right) / 2,
$$


as it is found in Case 1(iii). We use this solution in (B9) and get

$$
\begin{aligned}
& \int_{0}^{\rho} \frac{d \hat{\rho}}{\sqrt{\left(h(\hat{\rho})-f_{3}\right)\left(a h^{2}(\hat{\rho})+b h(\hat{\rho})+c\right)}}= \\
= & \int_{0}^{\rho} \frac{d \hat{\rho}}{\sqrt{A \cosh ^{3}\left(\sqrt{\kappa} \hat{\rho}+C_{1}\right)+B \cosh ^{2}\left(\sqrt{\kappa} \hat{\rho}+C_{1}\right)+C \cosh \left(\sqrt{\kappa} \hat{\rho}+C_{1}\right)+D}} \\
= & \pm \sqrt{\mu} \xi+C_{2},
\end{aligned}
$$

where $C_{2}$ is a constant and

$$
A=a b_{1}^{3}, B=b_{1}^{2}\left(3 a a_{1}+b-f_{3}\right), C=b_{1}\left(3 a a_{1}^{2}-2 a a_{1} f_{3}+2 b a_{1}-f_{3} b+c\right),
$$

and $D=\left(a_{1}-f_{3}\right)\left(a_{1}^{2} a+b a_{1}+c\right)$.

(iii) Take

$$
\begin{aligned}
& \left(\frac{d f}{d \rho}\right)^{2}=\kappa\left(f-f_{1}\right), \\
& \left(\frac{d \rho}{d \xi}\right)^{2}=\mu\left(f-f_{2}\right)\left(f-f_{3}\right)\left(a f^{2}+b f+c\right),
\end{aligned}
$$

where $\kappa \mu=\alpha$. Consider Equation (B12). It has a solution

$$
f=h(\rho)=\left( \pm \frac{\sqrt{\kappa}}{2} \rho+C_{1}\right)^{2}+f_{1}, \quad C_{1} \text { constant, }
$$

as it is obtained in Case 1(iv). We use this result in Eq. (B13)

$$
\begin{aligned}
& \int_{0}^{\rho} \frac{d \hat{\rho}}{\sqrt{\left(h(\hat{\rho})-f_{2}\right)\left(h(\hat{\rho})-f_{3}\right)\left(a h^{2}(\hat{\rho})+b h(\hat{\rho})+c\right)}}= \\
& =\int_{0}^{\rho} \frac{d \hat{\rho}}{\sqrt{\left(\frac{\kappa}{4} \hat{\rho}^{2} \pm \sqrt{\kappa} C_{1} \hat{\rho}+A\right)\left(\frac{\kappa}{4} \hat{\rho}^{2} \pm \sqrt{\kappa} C_{1} \hat{\rho}+B\right)\left(\frac{a \kappa^{2}}{16} \hat{\rho}^{4} \pm \frac{a \kappa^{3 / 2}}{2} C_{1} \hat{\rho}^{3}+C \hat{\rho}^{2}+D \hat{\rho}+E\right)}} \\
& = \pm \sqrt{\mu} \xi+C_{2},
\end{aligned}
$$

where $C_{2}$ is a constant and

$$
A=C_{1}^{2}+f_{1}-f_{2}, B=C_{1}^{2}+f_{1}-f_{3}, C=\kappa\left(b+6 a C_{1}^{2}+2 a f_{1}\right) / 4
$$

and

$$
D= \pm \sqrt{\kappa} C_{1}\left(2 a f_{1}+2 a C_{1}^{2}+b\right), E=\left(f_{1}+C_{1}^{2}\right)\left(a f_{1}+a C_{1}^{2}+b\right)+c .
$$

(iv) Take

$$
\begin{aligned}
& \left(\frac{d f}{d \rho}\right)^{2}=\kappa\left(a f^{2}+b f+c\right), \\
& \left(\frac{d \rho}{d \xi}\right)^{2}=\mu\left(f-f_{1}\right)\left(f-f_{2}\right)\left(f-f_{3}\right),
\end{aligned}
$$

where $\kappa \mu=\alpha$. Consider the first equation above. We have

$$
\int_{0}^{f} \frac{d \hat{f}}{\sqrt{a} \sqrt{\left(\frac{\hat{f}+\frac{b}{2 a}}{W}\right)^{2}+1}}= \pm \sqrt{\kappa} \rho+C_{1},
$$

where $W=\sqrt{4 a c-b^{2}} / 2 a$. Let $\left(f+\frac{b}{2 a}\right) / W=\tan \theta$. Hence the above equality becomes

$$
\int_{0}^{\theta} \sec \hat{\theta} d \hat{\theta}=\sqrt{a}\left( \pm \sqrt{\kappa} \rho+C_{1}\right)
$$

which gives

$$
\ln |\sec \theta+\tan \theta|=\sqrt{a}\left( \pm \sqrt{\kappa} \rho+C_{1}\right) .
$$


Hence after some simplifications and taking the integration constant zero, we get

$$
f=h(\rho)= \pm \sqrt{4 a c-b^{2}} \sinh (\sqrt{a \kappa} \rho) / 2 a .
$$

We use this result in (B17),

$$
\begin{aligned}
& \int_{0}^{\rho} \frac{d \hat{\rho}}{\sqrt{\left(h(\hat{\rho})-f_{1}\right)\left(h(\hat{\rho})-f_{2}\right)\left(h(\hat{\rho})-f_{3}\right)}}= \\
= & \int_{0}^{\rho} \frac{d \hat{\rho}}{\sqrt{A \sinh ^{3}(\sqrt{a \kappa} \hat{\rho})+B \sinh ^{2}(\sqrt{a \kappa} \hat{\rho})+C \sinh (\sqrt{a \kappa} \hat{\rho})+D}} \\
= & \pm \sqrt{\mu} \xi+C_{2},
\end{aligned}
$$

where $C_{2}$ is a constant and

$$
A= \pm\left(4 a c-b^{2}\right)^{-3 / 2} / 8 a^{3}, B=\left(b^{2}-4 a c\right)\left(f_{1}+f_{2}+f_{3}\right) / 4 a^{2},
$$

and

$$
C= \pm \sqrt{4 a c-b^{2}}\left(f_{1} f_{3}+f_{2} f_{3}+f_{1} f_{2}\right) / 2 a, D=-f_{1} f_{2} f_{3} .
$$

Case 3. If (9) has just one real root, we can write it in the form

$$
\left(f^{\prime}\right)^{2}=\alpha\left(f-f_{1}\right)\left(f^{4}+a_{3} f^{3}+a_{2} f^{2}+a_{1} f+a_{0}\right),
$$

where $f_{1}$ is the zero of the polynomial function $P_{5}(f)$ of degree five and the constants $a_{i}, i=$ $0,1,2,3$ are so that $f^{4}+a_{3} f^{3}+a_{2} f^{2}+a_{1} f+a_{0} \neq 0$ for real $f$.

Now define a new function $\rho(\xi)$, so that $f=f(\rho(\xi))$. Hence (B20) becomes

$$
\left(f^{\prime}\right)^{2}=\left(\frac{d f}{d \rho}\right)^{2}\left(\frac{d \rho}{d \xi}\right)^{2}=\alpha\left(f-f_{1}\right)\left(f^{4}+a_{3} f^{3}+a_{2} f^{2}+a_{1} f+a_{0}\right) .
$$

(i) Take

$$
\begin{aligned}
& \left(\frac{d f}{d \rho}\right)^{2}=\kappa\left(f-f_{1}\right), \\
& \left(\frac{d \rho}{d \xi}\right)^{2}=\mu\left(f^{4}+a_{3} f^{3}+a_{2} f^{2}+a_{1} f+a_{0}\right),
\end{aligned}
$$

where $\kappa \mu=\alpha$. Consider the first equation above. It has a solution

$$
f=h(\rho)=\left( \pm \frac{\sqrt{\kappa}}{2} \rho+C_{1}\right)^{2}+f_{1}, \quad C_{1} \quad \text { constant, }
$$

as it is obtained in Case 1(iv). We use this result in (B23) and get

$$
\int_{0}^{\rho} \frac{d \hat{\rho}}{\sqrt{h^{4}(\hat{\rho})+a_{3} h^{3}(\hat{\rho})+a_{2} h^{2}(\hat{\rho})+a_{1} h(\hat{\rho})+a_{0}}}= \pm \sqrt{\mu} \xi+C_{2},
$$

where $C_{2}$ is a constant. Here we do not get a simpler expression than the original equation. Hence it is meaningless to use the method of factorization of the polynomial for this case.

(ii) Take

$$
\begin{aligned}
& \left(\frac{d f}{d \rho}\right)^{2}=\kappa\left(f^{4}+a_{3} f^{3}+a_{2} f^{2}+a_{1} f+a_{0}\right), \\
& \left(\frac{d \rho}{d \xi}\right)^{2}=\mu\left(f-f_{1}\right),
\end{aligned}
$$

where $\kappa \mu=\alpha$. Here for simplicity, we will use the form $f^{4}+b f^{2}+c$ instead of the polynomial function of degree four above. Since $f^{4}+b f^{2}+c=\left(f^{2}+b / 2\right)^{2}-b^{2} / 4+c$, we will take $c>b^{2} / 4$ to get an irreducible polynomial. If $c=b^{2} / 4$, then take $b>0$. From $\left(\frac{d f}{d \rho}\right)^{2}=\kappa\left(f^{4}+b f^{2}+c\right)$,

$$
\int_{0}^{f} \frac{d \hat{f}}{\sqrt{\hat{f}^{4}+b \hat{f}^{2}+c}}= \pm \sqrt{\kappa} \rho+C_{1}, \quad C_{1} \quad \text { constant. }
$$


We have

$$
f(\rho)=\frac{\sqrt{2 c}}{\sqrt{-b+\sqrt{-4 c+b^{2}}}} \operatorname{sn}\left((\sqrt{2} / 2)\left(C_{1} \pm \sqrt{\kappa} \rho\right) \sqrt{-b+\sqrt{-4 c+b^{2}}}, k\right),
$$

where $k=\frac{\sqrt{2}}{2} \sqrt{\frac{-2 c+b^{2}+b \sqrt{-4 c+b^{2}}}{c}}$. Then we use this function $f$ in (B27) and get

$$
\int_{0}^{\rho} \frac{\sqrt{A} d \hat{\rho}}{\sqrt{\sqrt{2 c} \operatorname{sn}\left((A \sqrt{2} / 2)\left(C_{1} \pm \sqrt{\kappa} \rho\right), k\right)-A f_{1}}}= \pm \sqrt{\mu} \xi+C_{2}, \quad C_{2} \quad \text { constant, }
$$

where $A=\sqrt{-b+\sqrt{-4 c+b^{2}}}$.

Now take $c=b^{2} / 4$ with $b>0$. In this case, we have

$$
\int_{0}^{f} \frac{d \hat{f}}{\hat{f}^{2}+b / 2}= \pm \sqrt{\kappa} \rho+C_{1}, \quad C_{1} \quad \text { constant }
$$

which gives the solution

$$
f=h(\rho)= \pm \frac{\sqrt{2 b}}{2} \tan \left(\frac{\sqrt{2 b}}{2}\left(\sqrt{\kappa} \rho+\tilde{C}_{1}\right)\right) .
$$

Now use Equation (B27). We have

$$
\int_{0}^{\rho} \frac{d \hat{\rho}}{\sqrt{ \pm \frac{\sqrt{2 b}}{2} \tan \left(\frac{\sqrt{2 b}}{2}\left(\sqrt{\kappa} \hat{\rho}+\tilde{C}_{1}\right)\right)-f_{1}}}= \pm \sqrt{\mu} \xi+C_{2},
$$

where $C_{2}$ is a constant. Solving the above equation gives

$$
\frac{ \pm 4}{\sqrt{\kappa} b\left(A_{2}^{2}+A_{3}^{2}\right) A_{3}}\left\{\frac{\sqrt{2 b}}{4} A_{3}^{2} \ln \left[\frac{\left(A_{1}-A_{2}\right)^{2}+A_{3}^{2}}{\left(A_{1}+A_{2}\right)^{2}+A_{3}^{2}}\right]-b \arctan \left(\frac{2 A_{1} A_{3}}{A_{3}^{2}-A_{1}^{2}+A_{2}^{2}}\right)\right\}= \pm \sqrt{\mu} \xi+C_{2},
$$

where

$$
A_{1}=\sqrt{ \pm 2 \sqrt{2 b} \tan \left(\frac{\sqrt{2 b}}{2}\left(\sqrt{\kappa} \rho+\tilde{C}_{1}\right)\right)-4 f_{1}}, A_{2}=\sqrt{\sqrt{4 f_{1}^{2}+2 b}-2 f_{1}},
$$

and $A_{3}=\sqrt{\sqrt{4 f_{1}^{2}+2 b}+2 f_{1}}$.

${ }^{1}$ Gürses, M. and Karasu, A., "Degenerate Svinolupov systems,” Phys. Lett. A 214, 21-26 (1996).

${ }^{2}$ Gürses, M. and Karasu, A., "Integrable coupled KdV systems," J. Math. Phys. 39, 2103-2111 (1998).

${ }^{3}$ Alonso, L. M., "Schrödinger spectral problems with energy-dependent potentials as sources of nonlinear Hamiltonian evolution equations," J. Math. Phys. 21, 2342-2349 (1980).

${ }^{4}$ Antonowicz, M. and Fordy, A. P., "A family of completely integrable multi-Hamiltonian systems," Phys. Lett. A 122, 95-99 (1987).

5 Antonowicz, M. and Fordy, A. P., "Coupled KdV equations with multi-Hamiltonian structures,” Physica D 28, 345-357 (1987).

${ }^{6}$ Antonowicz, M. and Fordy, A. P., "Factorization of energy-dependent Schrödinger operators: Miura maps and modified systems," Commun. Math. Phys. 124, 465-486 (1989).

${ }^{7}$ Gürses, M., "Integrable hierarchy of degenerate coupled KdV equations" (unpublished), e-print arXiv:1301.4075.

${ }^{8}$ Gürses, M. and Pekcan, A., "Traveling wave solutions of the degenerate coupled Korteweg-de Vries equation," J. Math. Phys. 55, 091501 (2014).

${ }^{9}$ Bradburry, T. C., Theoretical Mechanics (R. E. Krieger Publishing Co., Malabar, FL, 1981).

${ }^{10}$ Tchebichef, M. P., "L’intégration des différentielles irrationnelles," J. Math. Pures Appl. 18, 87-111 (1853).

${ }^{11}$ Chen, S., Gibbons, G. W., and Yang, Y., "Friedmann's equations in all dimensions and Chebyshev's theorem," J. Cosmol. Astropart. Phys. 12, 035 (2014).

${ }^{12}$ Chen, S., Gibbons, G. W., and Yang, Y., "Explicit integration of Friedmann's equation with nonlinear equations of state," J. Cosm. and Astropart. Phys. 2015 (2015). 